Scientific Paper

\title{
Effects of detection system parameters on cross-correlations between MUAPs generated from parallel and inclined muscle fibres
}

\author{
Noureddine MESSAOUDI ${ }^{\mathrm{a}, \mathrm{b},}$, Raïs El'hadi BEKKA ${ }^{\mathrm{b}}$, Samia BELKACEM ${ }^{\mathrm{a}}$ \\ ${ }^{a}$ Université de Boumerdes, Faculty of Technology, Department Engineering of Electrical Systems, 35000 Boumerdes, Algeria \\ ${ }^{b}$ Université de Sétif 1, Faculty of Technology, Department of Electronics, LIS Laboratory, 19000 Sétif, Algeria \\ *E-mail address: n.messaoudi@univ-boumerdes.dz
}

\begin{abstract}
The aim of this study was to investigate the effects of inter-electrode distance (IED), electrode radius (ER) and electrodes configurations on cross-correlation coefficient (CC) between motor unit action potentials (MUAPs) generated in a motor unit (MU) of parallel fibres and in a MU of inclined fibres with respect to the detection system. The fibres inclination angle (FIA) varied from $0^{\circ}$ to $180^{\circ}$ by a step of $5^{\circ}$. Six spatial filters (the longitudinal single differential (LSD), longitudinal double differential (LDD), bi-transversal double differential (BiTDD), normal double differential (NDD), an inverse binomial filter of order two (IB2) and maximum kurtosis filter (MKF)), three values of IED and three values of ER were considered.

A cylindrical multilayer volume conductor constituted by bone, muscle, fat and skin layers was used to simulate the MUAPs.

The cross-correlation coefficient analysis showed that with the increase of the FIA, the pairs of MUAPs detected by the IB2 system were more correlated than those detected by the five other systems. For each FIA, the findings also showed that the MUAPs pairs detected by BiTDD, NDD, IB2 and MKF systems were more correlated with smaller IEDs than with larger ones, while inverse results were found with the LSD and LDD systems. In addition, the pairs of MUAPs detected by the LDD, BiTDD, IB2 and MKF systems were more correlated with large ERs than with smaller ones. However, inverse results were found with the LSD and NDD systems.
\end{abstract}

Key words: cross-correlation; electrodes; electromyography; fibres; inclination.

\section{Introduction}

The anatomy of muscles is generally complex and the fibres have several inclination angles in the muscle. ${ }^{1,2}$ The shape and amplitude of the single fibre action potential (SFAP) change with fibre inclination. The characteristics of the motor unit action potential (MUAP) and the surface electromyography (EMG) signals generated by muscles with inclined fibres depend on the orientation of the recording surface electrodes relative to the direction of the muscle fibres. ${ }^{1,3}$

On one hand, since the human body contains muscles with inclined fibres (case of the pinnate, bi-pinnate and multipinnate muscles ${ }^{2}$ ), so it is important to compare between surface EMG signals generated by muscles with fibres parallel to the arrangement of the electrodes and those generated by muscles with inclined fibres with respect to the electrodes arrangement. On the other hand, practically it is difficult to put all the electrodes in parallel with all the fibres of a muscle. Therefore, it is important to estimate the effect of the inclination of the fibres relative to the electrodes.

In our study, misalignment between fibres and electrodes is in a plane parallel to the skin surface. Moreover, with this type of muscles (in this study, the volume conductor model that we used is a cylindrical multilayer which is adapted to the limb muscle. Moreover, the inclination of the muscle fibres with respect to the electrodes arrangement is achieved by the rotation of the detection system on muscle fibres parallel to the skin surface) it is always important to consider the effect of each physiological and/or non-physiological parameter on the EMG signal in function of the angle between the fibres orientation and the arrangement of the electrodes. In the case of this type of muscle, surface potentials detected at different electrode locations along fibre direction alter shape and the system is not space-invariant in the direction of propagation of the intracellular action potentials (IAP). ${ }^{2}$

It was shown that the amplitude and the shape of surface EMG (sEMG) signals are highly affected by physiological and non-physiological parameters. ${ }^{4-6}$ However, in these studies, the effect of each of these parameters on the amplitude and spectral contents of the sEMG signal was assessed by fixing all other parameters. In the case of inclined muscle fibres, it is important to analyse the effects of anatomical, physical and detection system parameters according to the angle between the

(C) 2021 Noureddine Messaoudi, Raïs El'hadi Bekka, Samia Belkacem. This is an open access article licensed under the Creative Commons AttributionNonCommercial-NoDerivs License (http://creativecommons.org/licenses/by-nc-nd/4.0/). 
longitudinal axis of the fibres and the orientation of the electrodes (The angle of inclination of the fibres relative to the electrodes is considered in a plane parallel to the skin). The effects on surface EMG signals of some anatomical, physical and detection system parameters and fibre inclination were addressed only in a few works. ${ }^{3,4}$ In these studies only a few angles were considered. Fuglevand et al., ${ }^{7}$ studied by simulation the effects of IED and electrode size on the shape of the detected MUAP in the case where the detection system is aligned parallel to the direction of the fibres. Farina et al., investigated the influence of IED and ER and other anatomical and physical parameters on the shape of surface SFAP by varying the FIA from $0^{\circ}$ to $45^{\circ}$ by a step of $5^{\circ}$, and showed that the SFAP features depended on anatomical, physical and detection system parameters. Teklemariam et al., ${ }^{3}$ studied the effect of IED on electrode orientation relative to the direction the fibres for five values of the FIA $\left(0^{\circ}, 10^{\circ}, 30^{\circ}, 60^{\circ}\right.$ and $\left.90^{\circ}\right)$, and pointed out that the effect of significant misalignment between the electrodes and the fibres could be reduced by increasing the IED.

Many one-dimensional and two-dimensional linear, ${ }^{8}$ non linear $^{9}$ and adaptive ${ }^{10}$ detection systems were proposed for sEMG signal detection. The behaviour of these systems with respect to the inclination of the fibres is mainly related to its degree of isotropy. The anisotropic systems are very sensitive to the orientation of the fibres and the almost isotropic and purely isotropic ones are respectively less sensitive and not sensitive to the inclination of the fibres. ${ }^{4}$

Many anatomical, physical and detection system parameters can influence the sEMG signal. ${ }^{4}$ In turn, these parameters can be influenced by the orientation of the electrodes with respect to the direction of the muscle fibres. ${ }^{3,4,7,11-12}$ The effects of IED, ER and electrodes configuration on the correlation coefficient between pairs of MUAP signals generated from parallel fibres $\left(\right.$ FIA $\left.=0^{\circ}\right)$ and inclined fibres (FIA $\left.\neq 0^{\circ}\right)$ were investigated in this paper. Three values of the IED and three values of ER were used for this purpose. The simulated surface MUAP signals were generated in a multilayer cylindrical volume conductor consisting of bone, muscle, fat and skin layers as described in ${ }^{13}$ and detected by LSD, LDD, BiTDD, NDD, IB2 and MKF systems. The inclination of the fibres in the plane parallel to the skin was simulated by rotating the detection system with respect to its centre. This inclination was got by varying the FIA which is a parameter of the detection system transfer function from $0^{\circ}$ to $180^{\circ}$ by a step of $5^{\circ} .^{14}$

\section{Methods}

\section{Simulation of the MUAP}

The motor unit action potential (MUAP) of a MU is the sum of the SFAPs belonging to this MU. ${ }^{15}$
The simulated surface SFAP was implemented in MATLAB according to the model proposed by Farina et al., ${ }^{14}$ by performing the following three steps:

a) The current density source is proportional to the second derivative of the intracellular action potential (IAP). This IAP was described analytically by Rosenfalck, $1969 .{ }^{16}$ For each muscle fibre, two IAPs are generated at the neuromuscular junction (NMJ), and then they propagate in two opposite directions towards the tendons where they extinguish. ${ }^{14}$

b) The volume conductor was described as a multilayered cylinder composed of bone, muscle, and skin. The radius of the bone (homogeneous and isotropic, conductivity $0.02 \mathrm{~S} / \mathrm{m}$ ) was $20 \mathrm{~mm}$, the thickness of the muscle (homogeneous and anisotropic, longitudinal conductivity $0.5 \mathrm{~S} / \mathrm{m}$ and transversal conductivity $0.1 \mathrm{~S} / \mathrm{m}$ ) was $26 \mathrm{~mm}$, the thickness of the fat (homogeneous and isotropic, its conductivity was set $0.05 \mathrm{~S} / \mathrm{m}$ ) was $3 \mathrm{~mm}$ and the thickness of the skin layer (homogeneous and isotropic, conductivity $1 \mathrm{~S} / \mathrm{m}$ ) was $1 \mathrm{~mm}$. The simulated muscle has an elliptical cross section $(20 \mathrm{~mm}, 30 \mathrm{~mm})$ containing one MU where the coordinates of its centre were uniformly selected. The distance between the centre of the elliptical muscle and the centre of the volume conductor (the centre of the bone layer) was $34 \mathrm{~mm}$ The depth of the MU was defined as the distance between its centre and the interface muscle-fat. ${ }^{17}$ Figure 1 shows a cross-sectional view of the model used to generate the surface MUAP signals. Figure 2 shows the $3 \mathrm{D}$ longitudinal view of the volume conductor when fibres are parallel to the skin surface (Figure 2a) and when they are misaligned with respect to the skin surface. The volume conductor was mathematically described by a transfer function in the 2D spatial frequency domains, as suggested by Farina et al. 2004. ${ }^{14}$ The number of fibres within the MU is linked to its diameter. In this study the diameter of the MU was $4 \mathrm{~mm}$ and the density of the fibres was $20 \mathrm{fibres} / \mathrm{mm}^{2} .{ }^{13}$ The average fibre length varies in the range [40-160 mm] (here, the average fibre length was set $80 \mathrm{~mm}$ and the standard deviation was set $1 \mathrm{~mm}) .{ }^{18,19}$ The NMJs and the ending tendons were uniformly distributed over a range of $5 \mathrm{~mm} .{ }^{17}$

c) The detection system is the combination of the spatial filter and shape and size of the electrodes. ${ }^{14}$ This system is composed of one- and two-dimensional spatial filters with circular electrodes shape. The following detection systems were investigated: the longitudinal single differential (LSD), longitudinal double differential (LDD), bi-transversal double differential (BiTDD), normal double differential, (NDD), inverse binomial of order two $(\mathrm{IB} 2)^{8}$ and the maximum kurtosis (MKF) filter. ${ }^{10}$ The masks of these six spatial filters and the position of the electrodes arrangement with respect to the muscle fibres direction are shown in Figure 3. 


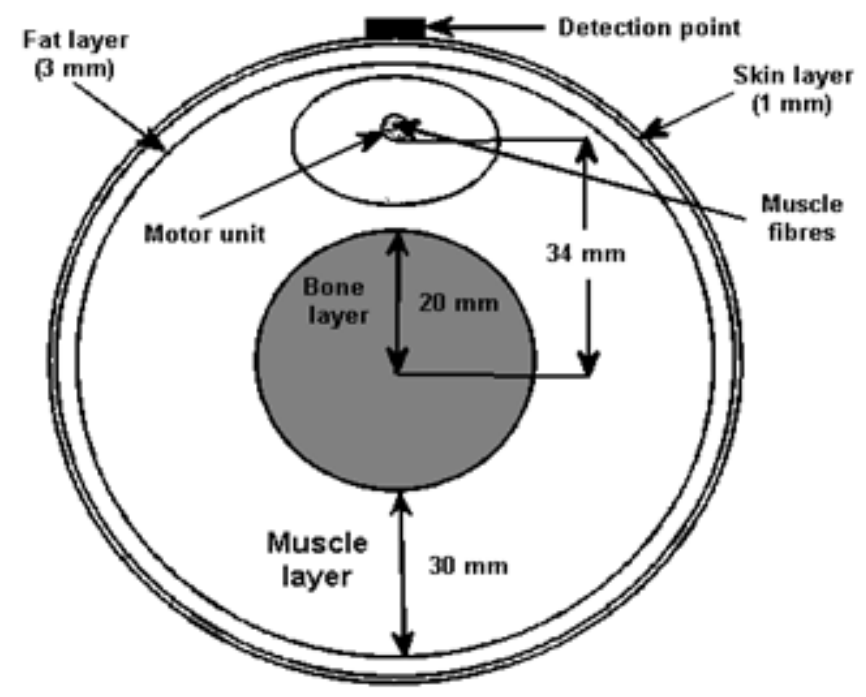

(a)
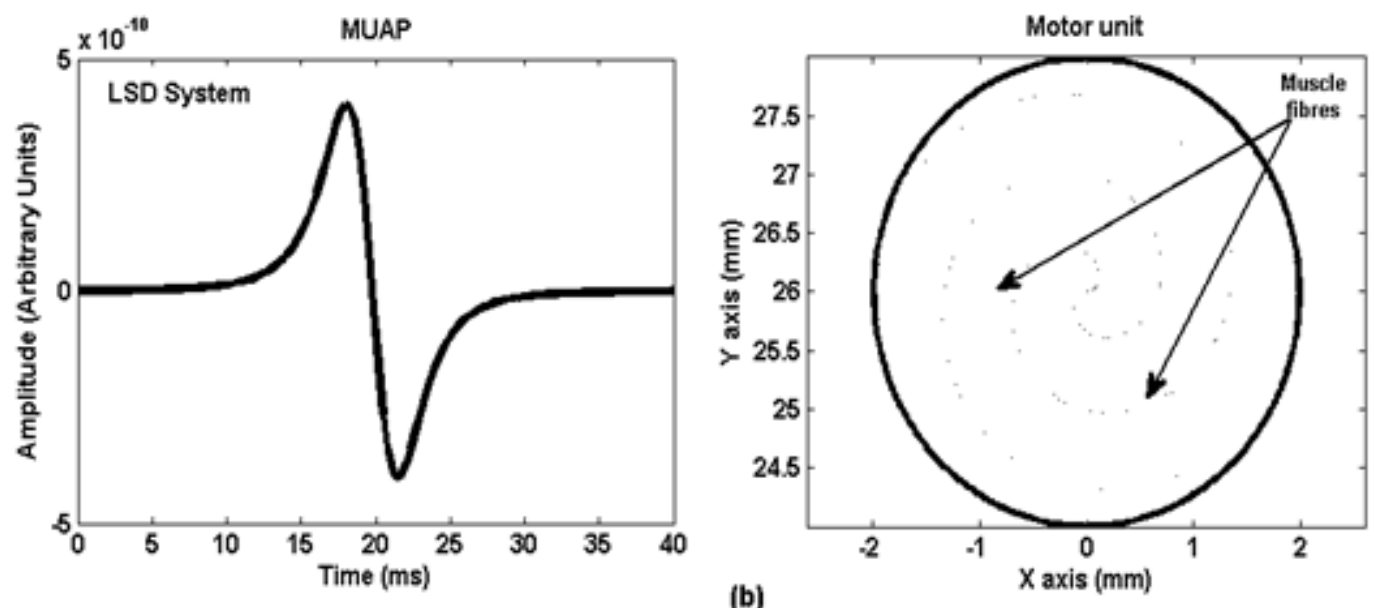

Figure 1. (a): The model investigated of the volume conductor. Within the elliptical muscle, there is a motor unit and within this motor unit there are 251 muscle fibres uniformly distributed ${ }^{11}$. (b): The motor unit (right side) and the generated MUAP (left side).
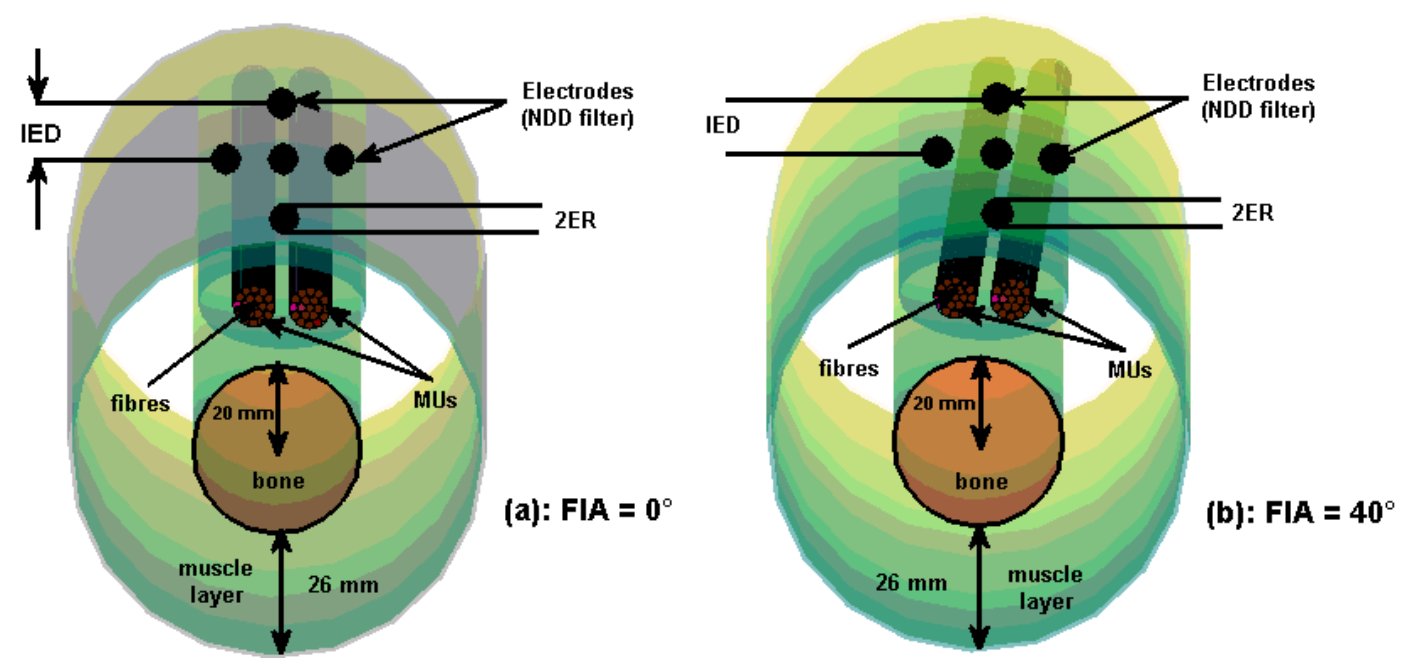

Figure 2. Longitudinal view of the volume conductor model used to simulate MUAPs generated from the two MUs. (a): case of fibres parallel $\left(\mathrm{FIA}=\mathbf{0}^{\circ}\right)$ to the electrodes arrangement. $(\mathrm{b})$ : case of fibres inclined $\left(\mathrm{FIA}=\mathbf{4 0}^{\circ}\right.$ ) with respect to the electrodes arrangement. 

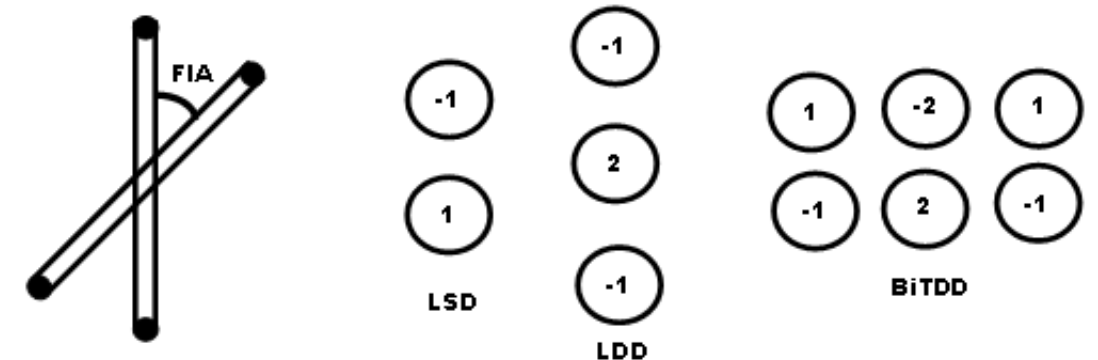

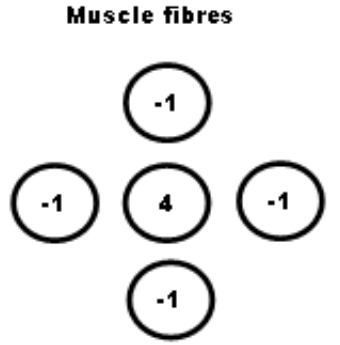

NDD

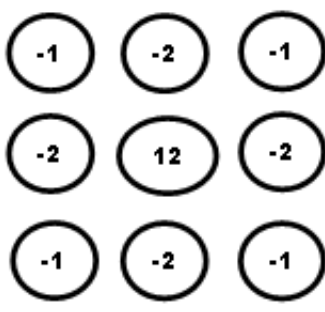

IB2

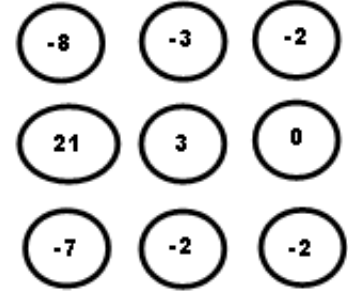

МKF

Figure 3. Arrangement of the electrodes configured in the seven investigated spatial filters with respect to the muscle fibres orientation. ${ }^{20}$

\section{Effects of IED, ER and electrodes configurations on CC}

The purpose of this study was to investigate the effects of the inter-electrode distance (IED), the electrode radius (ER) and the electrodes configuration on the cross-correlation coefficients (CCs) between MUAP signals generated by a MU of parallel fibres $\left(\mathrm{FIA}=0^{\circ}\right)$ to the detection system and a $\mathrm{MU}$ of inclined fibres with respect to the detection system (FIA $\neq$ $0^{\circ}$ ) when the FIA varied from $0^{\circ}$ to $180^{\circ}$ by a step of $5^{\circ}$. The generated MUAP signals were detected by the LSD, LDD, BiTDD (highly anisotropic), NDD and IB2 (almost isotropic) and MKF (adaptive and highly anisotropic) detection systems.

The Effect of IED was investigated for three values: 5, 10 and $20 \mathrm{~mm} .^{20}$ The effect of ER was investigated for three values: 4, 10 and $12 \mathrm{~mm} \cdot{ }^{20}$ For the analysis of the effect of a parameter with a given value on the cross-correlation coefficient, the fibres inclination angle (FIA) was varied from $0^{\circ}$ to $180^{\circ}$ in steps $5^{\circ}$. Consequently, 37 simulated MUAPs were generated and $37 \mathrm{CCs}$ were calculated i.e. the MUAP signal generated at FIA $=0^{\circ}$ was correlated with itself and was also correlated with the MUAPs signals generated when the FIA varied from $5^{\circ}$ to $180^{\circ}$ with a step of $5^{\circ}$.

\section{Results}

Figure 4 shows examples of simulated MUAP signals generated in the volume conductor previously described and detected by LSD (Figure 4a), LDD (Figure 4b), BiTDD (Figure 4c), NDD (Figure 4d), IB2 (Figure 4e) and MKF (Figure 4f) systems when the FIA varied from $0^{\circ}$ to $90^{\circ}$ in steps of $10^{\circ}$. It is clear that the LSD signal has the lowest amplitude and the MKF signal has the largest one. These results are due to the configuration of the electrodes of each system and to the appropriate weights assigned to them. The figure also shows that increasing the FIA decreased the amplitudes of the MUAP signals of LSD (Figure 4a), LDD (Figure 4b), BiTDD (Figure 4c) and the MKF (Figure 4f) systems and kept almost constant the amplitudes of the MUAP signals detected by the NDD (Figure 4d) and IB2 (Figure 4e) systems. Indeed the NDD and IB2 almost-isotropic filters compared to the LSD, LDD, BiTDD and MKF highlyanisotropic filters are less sensitive to the FIA as was underlined in ${ }^{21}$.

Table 1. Parameters used in the simulation of the results shown in Figure 4. ${ }^{17,18}$

\begin{tabular}{ccc}
\hline Parameter & Value & Description \\
\hline $\begin{array}{c}\text { Muscle cross section } \\
\text { conductivity) }\end{array}$ & $(30 \mathrm{~mm}, 20 \mathrm{~mm})$ & Elliptic \\
\hline $\begin{array}{c}\text { Muscle (thick, longitudinal } \\
\text { conductivity, transversal } \\
\text { conductivity) }\end{array}$ & $\begin{array}{c}(26 \mathrm{~mm}, 0.02 \mathrm{~S} / \mathrm{m}) \\
0.1 \mathrm{~S} / \mathrm{m})\end{array}$ & $\begin{array}{c}\text { Homogeneous } \\
\text { and isotropic }\end{array}$ \\
\hline $\begin{array}{c}\text { Fat (thick and conductivity) } \\
\text { Skin (thick and } \\
\text { conductivity) }\end{array}$ & $3 \mathrm{~mm}, 0.05 \mathrm{~S} / \mathrm{m}$ & $\begin{array}{c}\text { Homogeneous } \\
\text { and anisotropic }\end{array}$ \\
\hline Fibre diameter & $1 \mathrm{~mm}, 1 \mathrm{~S} / \mathrm{m}$ & $\begin{array}{c}\text { Homogeneous } \\
\text { and isotropic }\end{array}$ \\
\hline MFs lengths & $46 \mu \mathrm{m}$ & The same for all fibres \\
\hline MU diameter & {$[40-160 \mathrm{~mm}]$} & $\begin{array}{c}\text { Gaussian distribution } \\
\text { (mean = 80 mm, SD }= \\
1 \text { mm) }\end{array}$ \\
\hline Fibre distribution \\
within the MU
\end{tabular}



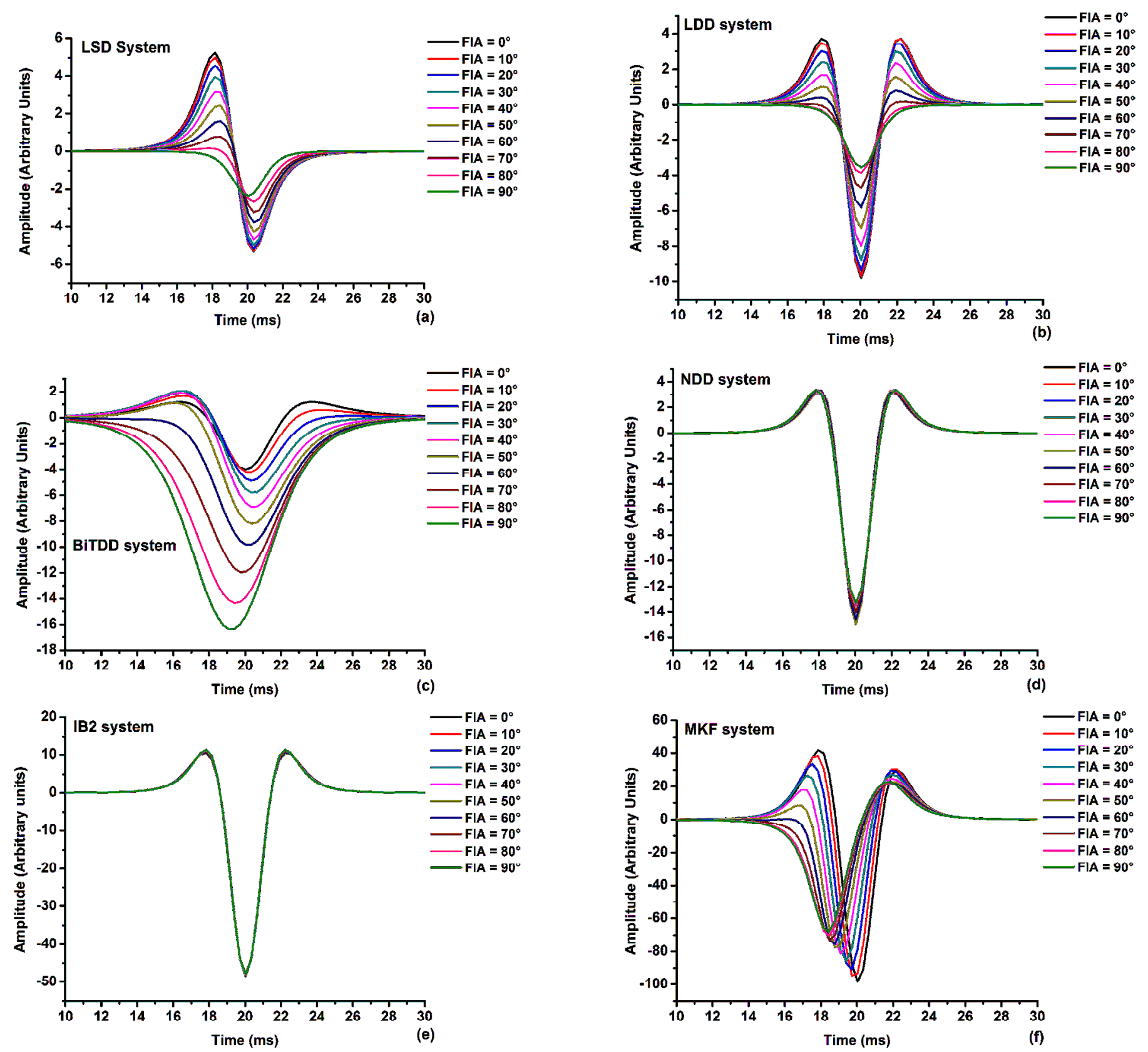

Figure 4. Simulated MUAP signals generated in a cylindrical multilayer volume conductor and detected by the six investigated detection systems when the FIA varied from $0^{\circ}$ to $90^{\circ}$ by a step of $10^{\circ}$. The IED was set $10 \mathrm{~mm}$. The radius of the electrodes was $4 \mathrm{~mm}$. The other parameters of simulation of these six signals are shown in Table 1.

Figures 5, 6 and 7 show the $\mathrm{CC}$ versus the FIA for three interelectrode distance values (Figure 5), three electrode radius values (Figure 6) and six detection systems (Figure 7). The results with respect to detection systems show that the $\mathrm{CC}$ curves have different shapes. Anisotropic spatial filters (LSD, LDD, BiTDD and MKF) provide CCs that are more sensitive to FIA than those obtained with the almost isotopic filters (NDD and IB2).

\section{Effect of the IED}

Figure 5 shows the IED effect on the CC of the pair of MUAP signals detected by LSD (Figure 5a), LDD (Figure 5b), BiTDD (Figure 5c), NDD (Figure 5d), IB2 (Figure 5e) and MKF (Figure 5f) systems. For each pair of MUAP signals, the first signal was generated from parallel fibres $\left(\right.$ FIA $=0^{\circ}$ ) and the second one was generated from inclined fibres when the FIA varied from $0^{\circ}$ to $180^{\circ}$ by a step of $5^{\circ}$. The analysis is made for three values of IED $(5,10$ and $20 \mathrm{~mm})$.

For example, Figure 5a shows that IED has a negligible influence on $\mathrm{CC}$ in the FIA range $\left[0^{\circ}-25^{\circ}\right]$ and a significant influence in the rest of the range in the case of LSD system, where the CC increases with decreasing IED. The CC of pair of MUAP signals detected by the IB2 (Figure 5e) and MKF (Figure 5f) systems depends on FIA and IED which decreases with the increase of FIA. These two figures show that the low value of the $\mathrm{CC}$ is obtained with IED $=5 \mathrm{~mm}$, and its high value is reached with IED $=20 \mathrm{~mm}$ and its intermediate value is got with IED $=10 \mathrm{~mm}$. 

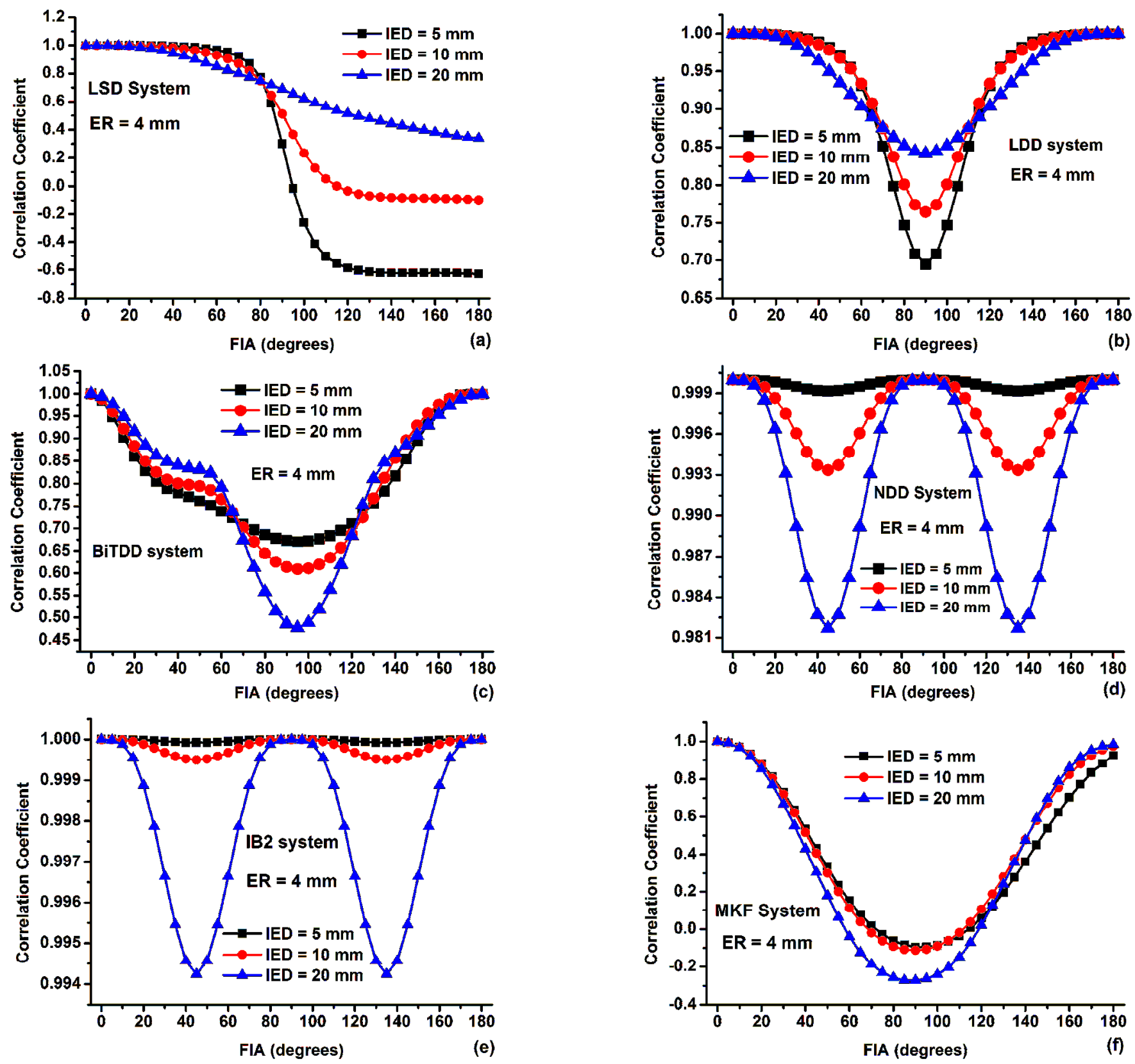

Figure 5. Effect of IED on the CCs of pairs of MUAP signals generated from parallel and inclined fibres and detected by LSD (a), LDD (b), BiTDD (c), NDD (d), IB2 (e) and MKF (f) systems. The radius of the circular electrodes was set 4 mm.

The IED effect was also investigated by the difference in percentage between the maximum (the best position of the electrodes with respect to the orientation of the fibres) and the minimum (to the worst position of the electrodes with respect to the orientation of the fibres) values of the CC (DMMCC) and the results are given in Table 2. This table shows that the increase in IED decreased the DMMCC \% value of LSD and LDD filters and increased the DMMCC \% value of BiTDD, NDD, IB2 and MKF filters. The results also show that the MKF system has the highest DMMCC values at IED $=10 \mathrm{~mm}$ and IED $=20 \mathrm{~mm}$ and that the LSD system has the highest DMMCC value at IED $=5 \mathrm{~mm}$ and the IB2 system has the lowest ones for all three IED values.
Table 2. The DMMCC of MUAP signals detected before and after the inclination of the fibres for three values of the IED. The ER was set $4 \mathrm{~mm}$.

\begin{tabular}{cccc}
\hline \hline & \multicolumn{3}{c}{ DMMCC \% } \\
\cline { 2 - 4 } Detection systems & \multicolumn{3}{c}{ IED (mm) } \\
\cline { 2 - 4 } & $\mathbf{5}$ & $\mathbf{1 0}$ & $\mathbf{2 0}$ \\
\cline { 2 - 4 } LSD & 162.78 & 109.751 & 65.998 \\
LDD & 30.35 & 23.58 & 15.84 \\
BiTDD & 32.93 & 39.11 & 52.27 \\
NDD & 0.085 & 0.665 & 1.83 \\
IB2 & 0.009 & 0.05 & 0.576 \\
MKF & 109.526 & 111.253 & 127.215 \\
\hline \hline
\end{tabular}



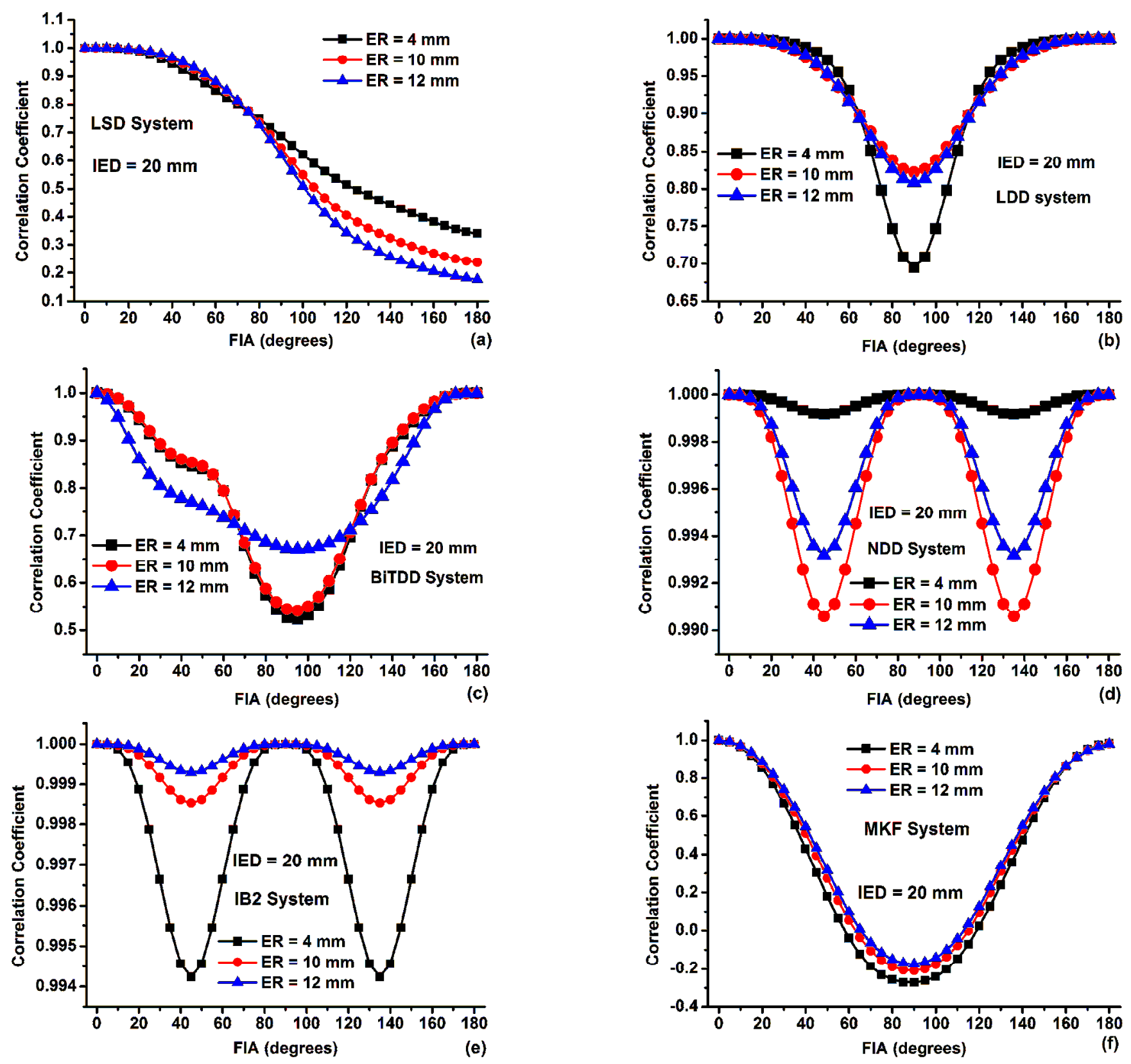

Figure. 6. Effect of the ER on CCs of MUAP signals generated from parallel and inclined fibres and detected by LSD (a), LDD (b), BiTDD (c), NDD (d), IB2 (e) and MKF (f) systems. The inter-electrode distance was $20 \mathrm{~mm}$.

\section{Effect of the ER}

The influence of electrode radius (ER) on $\mathrm{CC}$ values between the MUAP signals of two motor units for the six spatial filters was investigated for three electrodes radius values: $4 \mathrm{~mm}$, $10 \mathrm{~mm}$, and $12 \mathrm{~mm}$ The curves of CC as a function of FIA have the same behaviour as those showing the IED effect. For example, Figure 6a shows that increasing the radius of the electrode decreased the $\mathrm{CC}$ value between the pairs of MUAP signals detected by the LSD system for FIA varied from $90^{\circ}$ to $180^{\circ}$. However, in the interval $\left[0^{\circ}-90^{\circ}\right]$ of FIA, the effect of the ER on the $\mathrm{CC}$ is negligible. Figure 6e and Figure $6 \mathbf{f}$ show that for every FIA, when the ER increases, the CCs between MUAPs generated from parallel and inclined fibres increase.

The ER effect was examined by DMMCC \% as well and the results are given in Table 3. The results show that the increase in ER increased the DMMCC\% value of LSD and NDD filters and decreased those for LDD, BiTDD, IB2 and MKF filters.

Table 3. Effect of the ER on the DMMCC \% of the pairs of MUAP signals generated from parallel and inclined fibres. The IED was set $20 \mathrm{~mm}$.

\begin{tabular}{cccc}
\hline \hline & \multicolumn{3}{c}{$\boldsymbol{D M M C C} \%$} \\
\cline { 2 - 4 } Detection systems & \multicolumn{3}{c}{$\boldsymbol{E} \boldsymbol{R}(\mathbf{m m})$} \\
\cline { 2 - 4 } & $\mathbf{4}$ & $\mathbf{1 0}$ & $\mathbf{1 2}$ \\
\cline { 2 - 4 } LSD & 65.998 & 76.334 & 82.349 \\
LDD & 30.53 & 17.79 & 19.14 \\
BiTDD & 47.69 & 45.86 & 32.93 \\
NDD & 0.085 & 0.95 & 0.68 \\
IB2 & 0.576 & 0.146 & 0.071 \\
MKF & 127.21 & 120.896 & 117.519 \\
\hline \hline
\end{tabular}



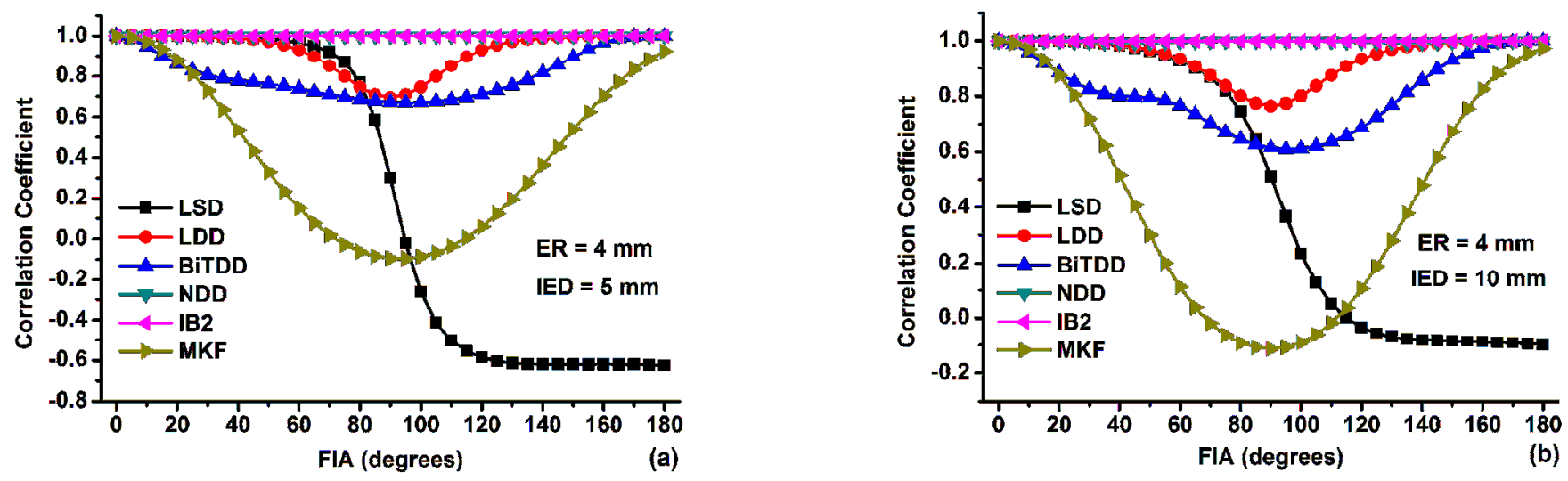

Figure. 7. Comparison of the effect of the FIA on CCs of MUAP signals generated from parallel and inclined fibres and detected by the six investigated systems.

In addition, with the three values of the ER, the highest values of the DMMCC\% are obtained with the MKF system and its smallest values are obtained with IB2 system. This means that the smaller DMMCC\% values show that the MUAP signals detected by the IB2 system are more correlated than those detected by the other five systems.

\section{Effect of the electrodes configuration (spatial filter)}

The effect of the configurations of spatial filters on the CC between two MUAP signals was studied for two values of the IED (5 and $10 \mathrm{~mm}$ ) and for ER $=4 \mathrm{~mm}$. Figure 7a and Figure $7 \mathbf{b}$ show the behaviours of the six investigated filters in function of the FIA and the IED. The two figures show that the FIA has almost no influence on CC between two MUAP signals detected by the NDD and IB2 systems. In general, despite the increase in FIA, the different pairs of MUAPs generated from parallel and inclined fibres are more correlated with the IB2 system than with the other systems.

\section{Discussion}

The inclination of the fibres with respect to the detection system was considered only in a few modelling studies and for a few FIA. ${ }^{3,4,14}$ However, the performance of any system depends on the positioning of the electrodes with respect to the muscle fibres, i.e. the FIA. ${ }^{3,4}$ In this study, we varied the FIA from $0^{\circ}$ to $180^{\circ}$ in steps of $5^{\circ}$ which corresponds to a half rotation of the detection system with respect to the direction of the muscle fibres.

Fuglevand et al., ${ }^{7}$ studied by simulation the effects of the IED and electrode size on the shape of the detected surface MUAPs in the case of parallel fibres. They showed that: (a) electrode size had a smaller effect on the detection depth of MUAPs, (b) the detection depth increased with increasing IED and (c) the frequency content of the action potentials decreased with increasing the electrode size and the radius of the motor unit. ${ }^{7}$ In our study, we have shown that the IED, ER and spatial filters have a great influence on the correlation coefficients between MUAPs generated by MU with parallel fibres and those generated by MU with inclined fibres (see Figures 5, 6 and 7 and Tables 2 and 3). In addition, our results show that for LSD and LDD systems, the correlation coefficients between MUAPs generated by parallel fibres and MUAPs generated by inclined fibres is greater for large IEDs and smaller for smaller IEDs. Inverse results were found with the BiTDD, NDD, IB2 and MKF systems (see Figure 5). For the effect of ER, we have shown that for the LDD, BiTDD, IB2 and MKF systems, the correlation coefficients between MUAPs generated by parallel fibres and MUAPs generated by inclined fibres are greater for large ERs and smaller for smaller ER. Inverse results were found with LSD and NDD systems (see Figure 6).

Teklemariam et al., $2016^{3}$ examined the effects of the IED and the electrode orientation relative to the fibres on the surface EMG signal when the FIA varied from $60^{\circ}$ to $90^{\circ}$ and showed that the effect of misalignment between the electrodes and the fibres could be reduced by increasing the IED (25-30 $\mathrm{mm}$ ), which attenuated the signal cancellation. The differences between the results obtained $\mathrm{in}^{3}$ and our results are as follows: (i) the results of ref. ${ }^{3}$ were based on a few values of the FIA (FIA varied from $60^{\circ}$ to $90^{\circ}$ ), while our results were based on the whole interval of variation of the FIA (FIA varied from $0^{\circ}$ to $180^{\circ}$ by a step of $5^{\circ}$ ), (ii) the detection system in ${ }^{3}$ was only the bipolar system, whereas in our study, three detection systems were used and compared, (iii) with the focus on the relationship between the effect of the IED and the inclination of the fibres, it was shown in $^{3}$ that the effect of the fibres inclination can be reduced by increasing the IED. The last result cannot be generalized to all detection systems. In fact, we have shown that MUAP signals generated from parallel and inclined muscle fibres are: (i) less correlated with a smaller IED $(5 \mathrm{~mm})$ and more correlated with a large IED $(20 \mathrm{~mm})$ in the case of LSD system. (ii) more correlated with a smaller IED $(5 \mathrm{~mm})$ and less correlated with large IED $(20 \mathrm{~mm})$ in the case of IB2 and MKF systems (see Table 2 and Figure 5).

Farina et al., 2002 $2^{4}$ studied the effect of the dimension of transversal and longitudinal bar electrodes in the case of double differential (DD) filter on the estimated conduction velocity 
(CV) with FIA $=30^{\circ}$. They showed that electrode size negligibly influenced $\mathrm{CV}$ estimation in the case of DD recordings, especially for large IEDs. In order to better analyze the effects of ER and electrode orientation, we used three $E R$ values and varied the FIA from $0^{\circ}$ to $180^{\circ}$. We showed that the MUAP signals generated from parallel and inclined fibres and detected by MKF and IB2 systems were more correlated with the large ER (12 $\mathrm{mm}$ of the radius of the circular electrode) than with the smaller one $(4 \mathrm{~mm}$ of the radius of the circular electrode). Inverse results were observed with the LSD system (see Figure 6).

The effects of fat layer thickness and effect of the maximum voluntary contraction (MVC) level on the CC of the sEMG signals generated from parallel and inclined fibres when the FIA varied from $0^{\circ}$ to $360^{\circ}$ by a step of $5^{\circ}$ and detected by the LSD, IB2 and MKF systems were examined in ${ }^{11}$. It was shown that the fat layer thickness negligibly influenced $\mathrm{CC}$ and that sEMG signals detected by LSD system were more correlated at a low level of MVC than at a high level of MVC, while the sEMG signals detected by IB2 and MKF systems have an inverse effect. In the present study, we found that the pairs of MUAP signals produced by a MU of parallel fibres to the detection system and a MU of inclined fibres relative to the detection system detected by IB2 and MKF systems were more correlated with a smaller IED than with a large one for each FIA. Inverse results were realized with the LSD system.

It was mentioned $\mathrm{in}^{22}$ that the IED should be in the range of 4-10 mm to provide at least a few samples for each MUAP, and large IEDs and improper electrode locations may lead to very deceptive results.

It was revealed $\mathrm{in}^{23}$ that the shapes of the recorded potentials (MUAP) depended on the properties and location of the electrodes and on the anatomy and physiology of the muscle fibres. It was also noted that the IED modified the spatial transfer function of the detection system and consequently influenced the signal properties. In this work, we showed that the pairs of MUAPs detected by the LSD system were more correlated with IED $=20 \mathrm{~mm}$ and less correlated with IED = $5 \mathrm{~mm}$ in the entire range of the FIA (see Figure 5a), and the pairs of MUAPs detected by the IB2 and MKF systems were more correlated with IED $=5 \mathrm{~mm}$ and less correlated with IED $=20 \mathrm{~mm}$ (see Figure 5e and Figure 5f, respectively) for all FIA values. But, we observed that IED negligibly influenced $\mathrm{CC}$ in the case of IB2 system (see Figure 5e).

The low-pass filtering effect due to the electrode size influenced the space and time features of the detected sEMG signal. $^{24}$ Therefore, smaller area electrodes are wanted. However, it was also pointed out that the reductions in impedance of the electrode relative to its contact area and noise were gained when the contact area of the electrode was increased. A trade-off between these two contradictory requirements can be reached by means of micro-needle or grooved surface electrodes in order that the smaller electrodes can have a fairly large contact area. It was shown that IED and
ER have a noticeable effect on the amplitude and spectral features of sEMG and can represent two confounding factors in sEMG measurements. ${ }^{24}$ We showed in the present study that the pairs of MUAPs detected by the IB2 (Figure 6e) and MKF (Figure 6f) systems were more correlated with ER $=12 \mathrm{~mm}$ and less correlated with $\mathrm{ER}=4 \mathrm{~mm}$ over the entire interval of FIA (Figure 6f).

The effect of the FIA on the degree of Gaussianity of surface EMG signals generated in a cylindrical multilayer volume conductor and detected by the three systems investigated here (LSD, IB2 and MKF) was assessed $\mathrm{in}^{25}$. The authors of the paper $^{25}$ showed that with the same detection system, the degree of Gaussianity of the EMG signal is highly influenced by the fibers inclination with respect to the electrode arrangement. Indeed, the classification of the studied detection systems according to the degree of Gaussianity of sEMG signals detected by them is different from a FIA interval to another. In relation to this work, for each value of the IED and ER, we showed that with the increase of the FIA, the pairs of MUAPs detected by the IB2 system were more correlated than those detected by LSD and MKF systems (see Figure 7a and 7f).

It was shown $\mathrm{in}^{21}$ that the NDD and IB2 systems are almost isotropic and that the LSD, LDD, BiTDD and MKF are anisotropic systems. Based on this important result, the results shown in Figures 5, 6 and 7 clearly show that with the NDD and IB2 systems the correlation coefficient has the same value at FIA $=0^{\circ}$ and at FIA $=90^{\circ}$ and at FIA $=180^{\circ}$. However, with LSD, LDD, BiTDD and MKF systems, the correlation coefficient is the same at FIA $=0^{\circ}$ and at FIA $=180^{\circ}$. This result is due to the arrangement of the electrodes in each detection system such as:

- For the LSD and LDD systems, the electrodes are arranged in only one direction and a $180^{\circ}$ rotation make the electrodes at the first arrangement (as at $0^{\circ}$ ).

- For the BiTDD and MKF systems, although these systems are bi-dimensional, but we have shown $\mathrm{in}^{21}$ that these two systems are anisotropic. Therefore, the variation of the correlation coefficient as a function of FIA is repeated every $180^{\circ}$.

- For the NDD and IB2 systems, since these two systems are two-dimensional and almost isotropic, ${ }^{21}$ the variation of the correlation coefficient as a function of FIA is repeated at every $90^{\circ}$.

\section{Limitations}

With the approach we proposed in this work, we cannot separate muscle fibres with different inclinations relative to the surface of the skin. Therefore, one cannot make an extension for simulating multiple simultaneous MUAPs generated by MUs of different inclinations and separating them. In perspective, we want to use an approach that allows separating motor units with inclined fibres from MUs with parallel fibres. 


\section{Conclusions}

A study of the cross-correlation coefficient (CC) between a pair of MUAP signals generated by a MU of muscle fibres parallel to the electrodes arrangement and a MU of inclined muscle fibres with respect to the electrodes arrangement was conducted in this paper by using simulated MUAPs signals. It focused on the influence of IED and ER on CC. Three detection systems were considered. The obtained results showed that the effects of IED and ER on CC depend on the detection system. But for the same detection system, each effect keeps the same behaviour for the different values assigned to the IED and ER. For each analysis parameter and for each value assigned to it, Tables $\mathbf{2}$ and $\mathbf{3}$ indicate that the DMMCCs of the IB2 system are the smallest and those of the
MKF system are the largest. This means that the MUAP signals detected by the IB2 system are strongly correlated despite the increase in the FIA. We showed that the increase in IED increased the DMMCC \% value of the MUAPs detected by the IB2 and MKF systems. However, the increase of the ER decreased the DMMCC \% value of the MUAPs detected by the IB2 and MKF systems. Inverse results were found with the LSD system. The lowest DMMCC\% values in the case of IB2 system implies that the MUAP signals detected by this system are more correlated than those detected by the other five systems. As a result, we recommended the IB2 system to record sEMG signals generated from muscles with inclined fibres.

\section{References}

1. Dimitrova NA, Dimitrov AG, Dimitrov GV, et al. Calculation of extracellular potentials produced by an inclined muscle fibre at a rectangular plate electrode. Med Eng Phys. 1999:21(8):583-588. https://doi.org/10.1016/s1350-4533(99)00087-9

2. Mesin L, Farina D. Simulation of surface EMG signals generated by muscle tissues with inhomogeneity due to fiber pinnation. IEEE Trans Biomed Eng. 2004:51(9):1521-1529. https://doi.org/10.1109/TBME.2004.827551

3. Teklemariam A, Hodson-Tole EF, Reeves ND, Costen NP, Cooper G, et al. A finite element model approach to determine the influence of electrode design and muscle architecture on myoelectric signal properties. PLoS-ONE. 2016:11(2):1-18. https://doi.org/10.1371/journal.pone.0148275

4. Farina D, Cescon C, Merletti R, et al. Influence of anatomical, physical, and detection-system parameters on surface EMG. Biol Cybern. 2002:86(6):445-456. https://doi.org/10.1007/s00422-002-0309-2

5. Farina D, Merletti R, Enoka RM, et al. The extraction of neural strategies from the surface EMG. J Appl Physiol. 2004:96(4):14861495. https://doi.org/10.1152/japplphysiol.01070.2003

6. Messaoudi N, Bekka RE. Simulated surface EMG signal as a function of physiological and non-physiological parameters: Analyze and interpretation. 2015: The Fourth International Conference on Electrical Engineering, ICEE2015, Boumerdes, Algeria, Proceedings, IEEE Xplore. https://doi.org/10.1109/INTEE.2015.7416801

7. Fuglevand A, Winter DA, Patla AE, Stashuk D, et al. Detection of motor unit action potentials with surface electrodes: influence of electrode size and spacing. Biol Cybern. 1992:67(2):143-153. https://doi.org/10.1007/BF00201021

8. Farina D, Arendt-Nielsen L, Merletti R, Indino B, Graven-Nielsen T, et al. Selectivity of spatial filters for surface EMG detection from the tibialis anterior muscle. IEEE Trans Biomed Eng. 2003:50(3):354-364. https://doi.org/10.1109/TBME.2003.808830

9. Zhou P, Suresh NL, Lowery MM, Rymer WZ, et al. Nonlinear spatial filtering of multichannel surface electromyogram signals during low force contractions. IEEE Trans Biomed Eng. 2009:56(7):1871-1879. https://doi.org/10.1109/TBME.2009.2017736

10. Östlund N, Yu J, Roeleveld K, Karlsson JS, et al. Adaptive spatial filtering of multichannel surface electromyogram signals. Med Biol Eng Comput. 2004:42(6):825-831. https://doi.org/10.1007/BF02345217

11. Messaoudi N, Bekka RE, Belkacem S, et al. Cross-Correlation coefficient as a means for estimating the effect of MVC level according to the fibres inclination', The Fifth International Conference on Electrical Engineering. 2017: ICEE2017, Boumerdes, Algeria, Proceedings, IEEE Xplore. https://doi.org/10.1109/ICEE-B.2017.8192166

12. Beck TW, Housh TJ, Cramer JT, Weir JP, et al. The effects of inter-electrode distance over the innervation zone and normalization on the electromyographic amplitude and mean power frequency versus concentric, eccentric, and isometric torque relationships for the vastus lateralis muscle. J Electromyogr Kinesiol. 2009:19(2): 219-231. https://doi.org/10.1016/j.jelekin.2007.07.007

13. Messaoudi N, Bekka RE. From single fibre action potential to surface electromyographic signal: A simulation study. Third International Conference, IWBBIO 2015, Granada, Spain, Proceedings, Part I, LNCS 9043, April 15-17, 2015:315-324. https://doi.org/10.1007/978-3-319-16483-0_32

14. Farina D, Mesin L, Simone M, Merletti R, et al. A surface EMG generation model with multilayer cylindrical description of the volume conductor. IEEE Trans Biomed Eng. 2004: 1(3): 415-426. https://doi.org/10.1109/TBME.2003.820998

15. Fuglevand AJ, Winter DA, Patla AE, et al. Models of recruitment and rate coding organisation in motor-unit pools. J Neurophysiol. 1993:70(6):2470-2488. https://doi.org/10.1152/jn.1993.70.6.2470 
16. Rosenfalck P. Intra and extracellular fields of active nerve and muscle fibres: A physico-mathematical analysis of different models. Acta Physiol Scand Suppl. 1969:321:1-168.

17. Messaoudi N, Bekka RE, Ravier P, Harba R, et al. Assessment of the non-Gaussianity and non-linearity levels of simulated sEMG signals on stationary segments. J Electromyog Kinesiol. 2017:32(1): 70-82. https://doi.org/10.1016/j.jelekin.2016.12.006

18. Keenan KG, Valero-Cuevas FJ. Experimentally valid predictions of muscle force and EMG in models of motor-unit function are most sensitive to neural properties. J. Neurophysiol. 2007:98(3):1581-1590. https://doi.org/10.1152/jn.00577.2007

19. Keenan KG, Farina D, Meyer FG, Merletti R, Enoka RM, et al. Sensitivity of the cross-correlation between simulated surface EMGs for two muscles to detect motor unit synchronization. J App Physiol. 2007:102:1193-1201. https://doi.org/10.1152/japplphysiol.00491.2006

20. Farina D, Merletti R. A novel approach for precise simulation of the EMG signal detected by surface electrodes. IEEE Trans Biomed Eng. 2001:48(6):637-646. https://doi.org/10.1109/10.923782

21. Messaoudi N, Bekka RE, Belkacem S, et al. Classification of the systems used in surface electromyographic signal detection according to the degree of isotropy. Adv Biomed Eng. 2018:7(1):107-116. https://doi.org/10.14326/abe.7.107

22. Barbero M, Rainoldi A, Merletti R, et al. Atlas of muscle innervation zones: understanding surface EMG and its applications. Springer, Italy 2012. https://doi.org/10.1007/978-88-470-2463-2

23. Merletti R, Farina D. (edts) Surface Electromyography: physiology, engineering and applications, IEEE Press / J Wiley, USA, May 2016.

24. Afsharipour B, Soedirdjo S, Merletti R, et al. Two-dimensional surface EMG: The effects of electrode size, interelectrode distance and image truncation. Biomed Sig Process Control. 2019: 49(1):298-307. https://doi.org/10.1016/j.bspc.2018.12.001

25. Messaoudi N, Bekka RE, Belkacem S, et al. Influence of fibers inclination on the degree of gaussianity of simulated surface EMG signals. ICBBT 2020, May 22-24, 2020, Xi'an, China. https://doi.org/ 10.1145/3405758.3405781 